\title{
Recent Progress on Spatial and Thematic Resolution in Landscape Ecology
}

\author{
Alex Mark Lechner ${ }^{1}$ • Jonathan R. Rhodes ${ }^{2,3}$
}

Published online: 12 August 2016

(C) Springer International Publishing AG 2016

\begin{abstract}
In this review we examine the past 5 years of landscape ecology research examining the effects of both spatial and thematic resolution on ecological analysis. While spatial resolution has been a topic of research for many decades, scale effects from thematic resolution have only recently been studied. Recent multi-scale analyses have demonstrated that the effects of thematic resolution on ecological analysis are comparable to spatial resolution and both spatial and thematic resolution interact. Additionally, spatial and thematic resolution can impact on multi-scale methods that are commonly used to identify the intrinsic scale - the scale at which ecological phenomena interact with the environment. A key aim of multi-scale analysis in landscape ecology is to characterise the scale-dependency of ecological processes, however, the interdisciplinary nature of landscape ecology means that research on scale has incorporated methods from a range of disciplines. These methods and concepts include: 1) multi-scale methods which characterise the sensitivity of an analysis to the modifiable areal unit problem, 2) spatial statistics for characterising the scale dependency of landscape patterns and identifying the optimal scale and 3) ecological theory describing intrinsic
\end{abstract}

This article is part of the Topical Collection on Scale-Measurement, Influence, and Integration

Alex Mark Lechner

a.lechner@uq.edu.au

1 Regional Water and Land Resources Program, Sustainable Minerals Institute, The University of Queensland, St Lucia, Brisbane, Queensland 4072, Australia

2 School of Geography, Planning and Environmental Management, The University of Queensland, Brisbane, QLD 4072, Australia

3 Centre for Biodiversity and Conservation Science, The University of Queensland, Brisbane, QLD 4072, Australia scales/domains of scales. We conclude by drawing on the existing literature to describe potential future areas of research to address the impacts of spatial and thematic resolution. We suggest further research on the effects of thematic resolution and conceptualising the scale continuum as multi-dimensional. We also identify mechanistic models and spatial statistics as a promising direction for future research.

Keywords Landscape ecology $\cdot$ Multi-scale $\cdot$ Scale $\cdot$ Spatial resolution $\cdot$ Spatial uncertainty $\cdot$ Thematic resolution

\section{Introduction}

Determining scale-dependencies of ecological responses to landscape patterns - in particular spatial scale - is a central question in landscape ecology and has been the focus of research for many decades [1-4]. Ecological processes are multi-scaled in nature and analysing them at inappropriate scales can result in the phenomenon remaining undetected due to a mismatch between the scale of the analysis and the scale of the ecological processes of interest $[3,5,6]$. This can potentially result in the derivation of spurious relationships and/or erroneous results $[7,8]$. Despite the recognition of the importance of scale, its impacts are not commonly addressed, partly due to the difficulties of conducting multiscale analysis [9].

There are numerous facets to scale in landscape ecology that affect the measurement of ecological responses and responses to management for conservation, two of which are the focus of this paper - spatial and thematic resolution. The first, spatial resolution, is probably one of the most studied aspect of scale research undertaken in the field of landscape ecology $[3,4,7]$, and related disciplines such as conservation biology $[10,11]$, spatial sciences $[12,13]$ and statistics $[14]$. While, the 
second aspect of scale, thematic resolution (also known as attribute precision [15] or categorical resolution [16]) which refers to the level of classification detail of the sampling units, is a relative newcomer to research in landscape ecology. Its effects on spatial analysis in landscape ecology have only recently been described in the ecological literature, with the earliest papers published in 2004 and 2007 [17-19].

In this review we look back on the past 5 years of landscape ecology research on the role of spatial and thematic resolution and evaluate the latest findings in light of the many decades of existing research. We first define spatial and thematic resolution in the context of an existing paradigm describing the domains of scale (observation, analysis and intrinsic scale). Next we summarise the effects of spatial and thematic resolution on ecological analysis and multi-scale analysis methods. We then focus on studies from the past 5 years, identifying examples of research that have made progress in characterising and addressing the effects of spatial and thematic resolution on ecological analysis. In particular we focus on studies which examined the effects of both spatial and thematic resolution. Drawing on these latest finding and more broadly from research in landscape ecology we conclude by suggesting future research directions for addressing spatial and thematic resolution.

\section{Definition and Effects of Spatial and Thematic Resolution, and Multi-Scale Methods}

\section{Dimensions of Scale and Definition of Spatial and Thematic Resolution}

Spatial resolution, often referred to as the grain size, is a product of several factors associated with the acquisition and processing of spatial data from the field or remote sensing [20-22]. In remote sensing, spatial resolution is primarily a product of the pixel size of the sensor or the object-scale for data produced through geographic object-based image analysis [22-26]. Pre- and post-processing of spatial data, commonly used for improving classification and geometric accuracy, also affects spatial resolution through: removing features below a minimum area (minimum mappable unit) [27, 28], the application of smoothing filters [29] and the characteristics of the remote sensing device [30].

Thematic resolution is a property of the ruleset used to describe a hierarchy of land cover classes, the criteria used to distinguish them, and the relationship between the classes. The Anderson classification scheme is a classic example of a land use/land cover classification hierarchy that has a hierarchy of different thematic resolutions [31]. The Anderson classification scheme contains four resolutions with each finer resolution class nested within a coarser resolution level class. For example, residential and industrial are nested within urban. Within the vegetation sciences thematic resolution can be considered as the hierarchical relationships between vegetation classification units where natural taxonomic units can be grouped, and/or regrouped from individual species, communities, to biogeographic regions [32]. In continuous data (i.e. temperature, rainfall) thematic resolution refers to how accurately environmental variability is measured [33] (e.g. what is the precision of the units measuring thematic differences).

Spatial and thematic resolution represent aspects of the three dimensions of scale [34] or three kinds of scale [2]. The first dimension, the observation/measurement scale, describes the size, shape, extent and distance between observational units used to sample a phenomenon. The second dimension, the analysis/modelling scale refers to the units used to analyse data and is an aggregate of the spatial units at the observation scale. When using remote sensing data, the observation scale is often the pixel size and the units of the analysis scale are patches in the landscape (or sometimes circular buffers around point locations), where each patch is composed of multiple pixels whose values are aggregated (e.g. percent vegetation cover for each patch).

The final dimension, the intrinsic scale, is the scale at which ecological phenomena interact with the environment and is an emergent property of an organism's response to its environment, which is measured indirectly at the observation scale(s) and/or analysis scale(s) [2, 29, 35]. In this paper we focus primarily on observation scale effects from changing spatial and thematic resolution in the context of GIS analysis using data derived from remote sensing. The combination of GIS analysis with remote sensing data represents one of the most common ways for representing and analysing data in landscape ecology [25, 36, 37].

\section{Effects on Ecological Analysis}

Multi-scale analyses are often used to test the sensitivity of a statistical analysis to variations in the spatial units [38, 39] such as in the case of the assessment of the modifiable areal unit problem (MAUP) [8] and the related change of support problem (COSP) [14]. The MAUP view of scale suggests that statistical relationships derived from areal units with different boundaries and levels of aggregation can confound the results of spatial statistical analyses [7, 40]. While the COSP is broader than the MAUP and refers to the effects of the types, size and shape of the spatial units used in spatial analysis, e.g. point, lines, areas and pixels [14, 34].

Changing spatial and thematic resolution have been shown to affect both the characterisation of landscapes and ecological analysis such as measuring land cover proportions [41-43], landscape metrics [19, 44, 45], graph-based connectivity metrics [46, 47], change detection [48] and statistical relationships between the landscape context and species distributions [49, 
50]. Spatial resolution has been demonstrated to affect statistical analysis such as inference about population mean, variation and statistical significance [43, 51-54].

Along with the effects of spatial and thematic resolution on analyses of ecological phenomenon that are considered purely from a statistical point of view or to test the sensitivity of an analysis to scale, multi-scale analysis with ecological data (i.e. species abundance, occurrence) are commonly used to identify intrinsic-scales associated with pattern-process relationships. The relationship of scale with ecological processes is described by related theories such as scale discontinuities [55, 56], domains of scale $[3,57]$ and hierarchy theory $[20]$ (see Nash et al. [56] for a summary).

\section{Multi-Scale Analysis Methods}

A common experimental design for assessing scale effects in landscape ecology is to aggregate pixels or alter the thematic resolution of one or more land cover maps and quantify changes to response variables, such as landscape metric values [6, 58-60]. Another common multi-scale analysis method used frequently in landscape ecology characterises multiscale species-environment relationships by altering the size of circular buffers/kernel around point locations at which ecological data is sampled [61-63], while leaving the spatial or thematic resolution constant. However, this type of multi-scale analysis is not an explicit focus of this paper as it does not involve altering the spatial and/or thematic resolution to identify scale effects (see $[63,64]$ for a comprehensive review).

In parallel to the ecological literature focusing on ecological pattern-process scale effects, the spatial sciences and, in particular the geostatistics/spatial statistics literature, have also addressed scale issues, but more from the perspective of quantifying the scale dependence of observed landscape patterns and finding the optimal spatial scale for mapping [35, 65-67]. These methods address the relationship between the sampling unit (observation scale) and the feature being measured (i.e. the relationship between a tree and a $30 \times 30 \mathrm{~m}$ pixel used to sample tree density) by factoring-in the spatial structure of the landscape such as clustering and directionality [67]. In ecology these methods have also been used to measure the statistical property of the spatial pattern of the landscape for assessing the relationship between spatial patterns and processes [67-69]. These techniques are also commonly used to address the COSP associated with mapping continuous spatial data (i.e. temperature, soil PH) with point data. Spectral decomposition techniques including wavelet analysis [70] and eigenvector approaches [71] represent another suite of statistical techniques that has been applied to ecological analysis to determine the key spatial scales at which underlying ecological processes act on species.

\section{Recent Progress (Post-2010) in Research on the Effects of Spatial and Thematic Resolution on Ecological Analysis}

Our review found that in the past 5 years (2010-2015) there has continued to be more research on spatial resolution than thematic resolution, with the number of publications on spatial resolution rising, while the number of publications on thematic resolution having stayed constant with an average of 6.8 publications per year, compared to 113.2 for spatial resolution (identified with a Scopus keyword search on 16/12/2015 (thematic or spatial resolution and ("scaling" OR "multi-scale"))). A recent study that reviewed all the studies published in landscape ecology using remote sensing data in 2007 found only $6 \%$ of articles addressed the impact of thematic resolution [25]. A keyword search examining studies that had assessed both aspects of resolution ("spatial resolution AND thematic resolution") identified a total of only 15 publications in the past 5 years (some of which were not ecological studies), indicating very little focus on dealing with both aspects of scale simultaneously.

In the past 5 years there have been a number of publications further developing the 'theory of scale' in the context of ecological analysis $[56,57,72]$ that are relevant to understanding and interpreting the effects of spatial and thematic resolution on ecological analyses. They build on existing theories that consider ecological phenomena to interact with or perceive the environment at relatively isolated, distinct scales or intrinsic scales defined by specific pattern-process relationships. For example, influences on foraging movement operate at fine-scales and movement for dispersal operates at coarser scales [73]. These theories suggest that along the scale continuum there are segments/domains which are invariant to scale and then transition zones or discontinuities at scales where it is not sensible to measure patterns [56,57]. It is within the context of these broader ecological concepts that the effects of spatial and thematic resolution are often addressed with multi-scale analysis in landscape ecology.

While it has been recognised for some time that spatial and thematic resolution impact on the measurement of ecological responses [17-19, 53, 54, 74], only recently have studies looked at both of these factors together. Several studies have compared the effects of using explanatory variables with a range of thematic and spatial resolutions in species distribution modelling and showed that they have a large effect on predictive accuracy [75, 76]. In particular Liang et al. [77] modelled three thematic and six spatial resolutions and assessed individual effects and interactions. They showed that both thematic and spatial resolutions affected model-based predictions of tree species distribution. 
For multi-scale analysis of landscape metrics, previous studies, before 2010, have tested multiple scaledependent factors such as extent and spatial resolution $[6,58,78]$, but only recently have studies explicitly tested interactions and effect sizes statistically [77, 79]. Studies examining the effects of scale on the calculation of landscape metrics using analysis of variance have shown statistically that factors interact [79, 80]. This means that not only do spatial and thematic resolution have main effects, but that the level of one will affect the level of another. Thus, studies that consider a single factor in isolation (e.g. pixel size) may produce different results when other factors are fixed at different levels.

In addition to the recognition that thematic and spatial resolution interact, affecting predictive modelling and the derivation of landscape metrics, they also affect other forms of multi-scale analysis. Ecological studies characterising an intrinsic scale infer that differences in correlation coefficients are related to the response of an ecological phenomenon to particular scales, and scales where there are high correlation coefficient values represents intrinsic scales [62, 81]. In most cases these forms of multi-scale analysis involve assessing the effects of analysis scale by changing the size of circular buffers around point locations at which ecological data are sampled for testing multi-scale species-environment relationships [64, 82, 83]. However, a recent study by Kendall et al. [50] using this multi-scale buffer analysis method showed that patterns of $\mathrm{r}^{2}$ values for a range of buffer sizes changed with thematic resolution and spatial resolution (defined by the minimum mappable unit). Additionally, Lechner et al. [29] using synthetic analysis showed that the highest $r^{2}$ values may incorrectly identify intrinsic scales identified with multi-scale buffer analyses as a result of changes in pixel size and applying a smoothing filter (which also reduces spatial resolution).

The recent studies described above have utilised relatively simple multi-scale analyses methods (often equivalent to a sensitivity analysis) for examining the effects of spatial and thematic resolution. While using spatial statistics for examining spatial resolution are very common in ecology [84-86], we only know of one example where these methods have been used to address the effects of thematic resolution [87]. Vicente et al. [87] provides an example of the use of spatial autocorrelation analysis (using Moran's $I$ and Geary's $C$ ) and statistical clustering to identify scales of spatial patterning of environmental factors for a range of thematic and spatial resolutions.

The differences between the types of multi-scale analyses that have been used in landscape ecology are nuanced and include a range of sometimes contradictory aims. The interdisciplinary nature of landscape ecology means that research on scale has incorporated methods from remote sensing, spatial statistics and ecological theory, with the objective of characterising the sensitivity of an analysis to scale (MAUP), address scale effects by identifying scaledependence and optimal scales, and explaining scale effects in terms of ecological processes by their intrinsic scales/ domains of scale (Fig. 1).

One of the key aims in the discipline of landscape ecology, which unites much of the research, is to characterise the scale-dependency of ecological processes. Figure 2a, b, c and d describe a classic conceptual diagram of the scale continuum [e.g. 9] which underpin scale theory in ecology. Although commonly the scale continuum is conceptualised for a single scale, it is in fact multi-dimensional and needs to be re-conceptualised as multi-dimensional, as studies identified in this review have shown, both thematic and spatial resolution interact and cannot be considered in isolation (Fig. 2e). Furthermore, each environmental variable used in an ecological analysis may be represented by multiple spatial datasets with a range of observation scales [64, 72], which each could be represented by multiple scale dimensions.

Thus, there is a need for a better understanding of the link between ecological scale theories, such as conceptualised in Fig. 2e, with multi-scale spatial analysis methods. Currently, there is no substantive difference in type of analysis conducted for studies testing for the presence of the MAUP (which indicate an analysis is not robust and perhaps spurious), to studies characterising the intrinsic scale. Crucial to deepening the understanding of the multiple dimensions of scale, is more research on the effects of thematic resolution and the combined effects of thematic and spatial resolution. Many studies comparing the influence of thematic resolution versus spatial

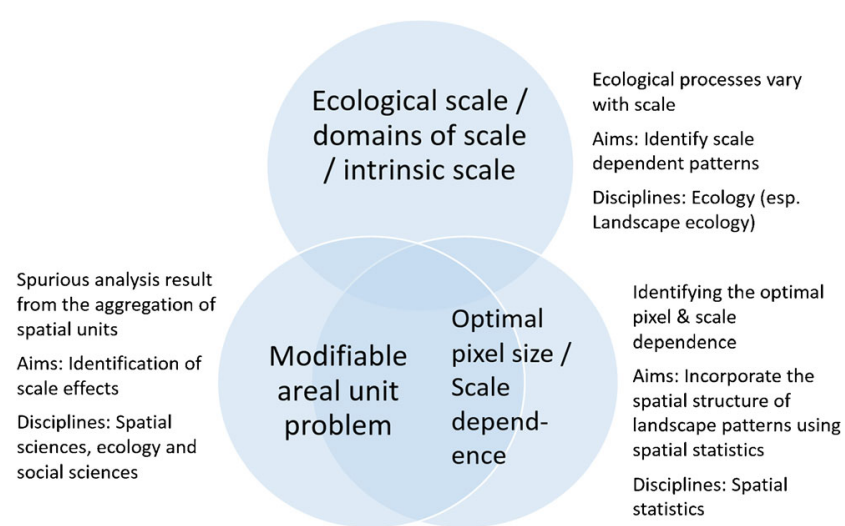

Fig. 1 Three different kinds of treatment of scale and the corresponding methods employed in landscape ecology that originate from a number of different disciplines, with a range of objectives. The bottom two circles in the Venn diagram are purposefully positioned closer together to reflect the fact that they represent very similar concepts and are often treated in the same way. Future solutions to scale may lie in integrating these different methods and concepts 

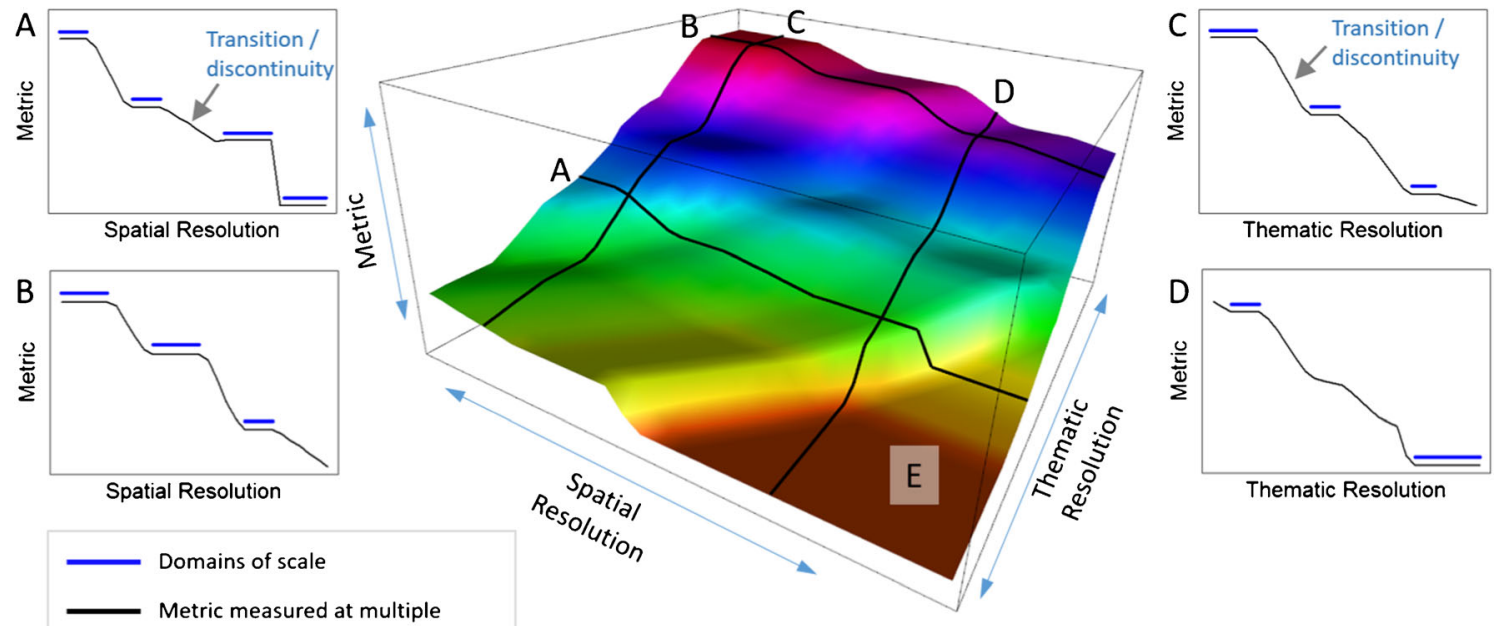

Spatial Resolution

Domains of scale

Metric measured at multiple

scales (such as species diversity)

Fig. 2 Panels a-d describe a common conceptualisation of the scale continuum in landscape ecology based on the relationship between scale (in this case spatial or thematic resolution) on the $\mathrm{x}$-axis and ecological metric values on the y-axis such as population viability, abundance and species richness. Scale (a-d) is conceptualised as having a number of domains identified by segments along the scale continuum (blue lines) at which the metric of interest is relatively constant. Commonly multi-scale analyses only consider a single aspect of scale (e.g. spatial or thematic resolution) at a time. In panel (e) we have represented the interaction of different aspects of scale as a two-dimensional surface where black lines across the surface represent analyses based on a single scale dimension $(\mathbf{a}, \mathbf{b}, \mathbf{c}, \mathbf{d})$ resolution show that thematic resolution has as a great, or greater influence on the results of ecological analyses than spatial resolution. For example, a recent study by Cushman and Landguth [33] assessing the impact of thematic resolution, pixel size and focal window size on landscape genetic analysis found that thematic resolution had the largest effect. Additionally, the relationship between studies assessing intrinsic scale and spatial statistics (i.e. geostatistical) methods are unclear, especially for thematic resolution which has received little attention in the ecological literature.

\section{Future Research Directions}

Common approaches to multi-scale analyses of spatial and thematic resolution that simply aggregate spatial data to identify relationships between ecological metrics and scale can be a blunt instrument for understanding scale-dependent ecological responses. One aspect that limits understanding of spatial and thematic resolution is a lack of integration of ecological processes into scale-dependent models. Scale-dependent relationships emerge from a myriad of ecological process operating at different scales [56, 64, 67] and generalisations about scale should consider these processes more explicitly. While, spatial statistics can be used to measure the statistical property of spatial patterns for multiple spatial predictors at multiple scales in order to identify the appropriate predictor to model the relationship between species' distributions and environmental variables [67]. Consequently, we suggest two ways forward for future research in this area: 1) utilising mechanistic models for capturing ecological processes [72, 88] and 2) integrating ecological theory/models with spatial statistics [87].

Using mechanistic models multi-scale processes can be captured explicitly by representing how organisms interact with landscape features at different scales. For example, species' foraging movements operate at finer spatial scales to dispersal and migratory movements and respond to different habitat components at each scales [89]. These phenomena can be accounted for by simultaneously modelling movement for foraging and dispersal processes that are explicitly represented by movement interactions with fine and coarse levels of spatial and thematic data aggregation respectively [73, 90]. The key advantage of using a more mechanistic approach is being explicit about the multi-scaled ecological processes that drive emergent scale-dependent ecological patterns and thereby allow for a more generalisable understanding of scale.

Recent advances in mechanistic and hierarchical statistical approaches will help to parameterise and develop these models from data [91]. However, approaches such as this will also require more explicit multi-scale characterisation of landscape features, which in part can be provided by multi-scale object-based image analysis methods with fine-scale spatial data to minimize the artificial boundaries associated with the pixels at multiple spatial resolutions [92].

While some ecological processes can be explicitly characterised with mechanistic models, an alternative approach or an approach to be used in parallel, is to characterise ecological patterns by their spatial structure using spatial statistics. Rather than explicitly representing landscape features and ecological processes using mechanistic methods as described above, spatial statistics can be used to summarise complex 
multi-scale relationships. These methods need to be developed to test multiple aspects of scale in a single analysis and aspects of scale that have received little attention such as thematic resolution. Vicente et al. [87] provides a promising example, how spatial autocorrelation analyses characterising multiple environmental factors - at a range of spatial and thematic resolutions - can be used to build predictors for ecological models (see [87, 93]). Such frameworks can potentially also incorporate data from mechanistic models as inputs into their analysis.

\section{Conclusions}

In the past 5 years there has been progress in addressing spatial and thematic resolution in landscape ecology, however, research on thematic resolution has lagged, even though its impacts are comparable to spatial resolution. Furthermore, research on the interactions between thematic and spatial resolution demonstrates that scale is even more complex than previously acknowledged and multi-dimensional. There are numerous methods for addressing scale issues arising from a range of disciplines that intersect with landscape ecology which require further consideration and definition. Studies which combine methods that explicitly account for the multi-dimensional nature of spatial and thematic resolution, using mechanistic models and/or spatial statistics represent a promising area of future research.

\begin{abstract}
Acknowledgements We thank the anonymous reviewers for their constructive feedback which has significantly improved the quality of the manuscript. This work was supported by funding from the Australian Research Council Centre of Excellence for Environmental Decisions.
\end{abstract}

\section{Compliance with Ethical Standards}

Conflict of Interest On behalf of all authors, the corresponding author states that there is no conflict of interest.

Human and Animal Rights and Informed Consent This article does not contain any studies with human or animal subjects performed by any of the authors.

\section{References}

1. Turner MG, Dale VH, Gardner RH. Predicting across scales: theory development and testing. Landsc Ecol. 1989;3:245-52.

2. Wu J, Li H. Concepts of scale and scaling. In: Wu J, Jones KB, Li $\mathrm{H}$, Louck OL, editors. Scaling uncertain analysis in ecology: methods and applications. Dordrecht: Springer; 2006. p. 3-15.

3. Wiens JA. Spatial scaling in ecology. Funct Ecol. 1989;385-97.

4. O'Neill RV, Hunsaker CT, Timmins SP, Jackson BL, Jones KB, Riitters $\mathrm{KH}$, et al. Scale problems in reporting landscape pattern at the regional scale. Landsc Ecol. 1996;11:169-80.
5. Levin SA. The problem of pattern and scale in ecology. Ecol Soc Am. 1992;73:1943-67.

6. Wu JG, Shen WJ, Sun WZ, Tueller PT. Empirical patterns of the effects of changing scale on landscape metrics. Landsc Ecol. 2002;17:761-82.

7. Jelinski D, Wu J. The modifiable areal unit problem and implications for landscape ecology. Landsc Ecol. 1996;11:129-40.

8. Openshaw S. The modifiable areal unit problem. Concepts Tech Mod Geogr. 1984;38:41.

9. Wheatley M, Johnson C. Factors limiting our understanding of ecological scale. Ecol Complex. 2009;6:150-9.

10. Lechner AM, Raymond CM, Adams VM, Polyakov M, Gordon A, Rhodes JR, et al. Characterizing spatial uncertainty when integrating social data in conservation planning. Conserv Biol. 2014;28: 1497-511.

11. Seo C, Thorne JH, Hannah L, Thuiller W. Scale effects in species distribution models: implications for conservation planning under climate change. Biol Lett. 2009;5:39-43.

12. Woodcock CE, Strahler AH. The factor of scale in remote-sensing. Remote Sens Environ. 1987;21:311-32.

13. Atkinson PM, Tate NJ. Spatial scale problems and geostatistical solutions: a review. Prof Geogr. 2000;52:607-23.

14. Gotway CA, Young LJ. Combining incompatible spatial data. J Am Stat Assoc. 2002;97:632-48.

15. Cunningham MA. Accuracy assessment of digitized and classified land cover data for wildlife habitat. Landsc Urban Plan. 2006;78: 217-28.

16. Franklin J, Woodcock CE. Multiscale vegetation data fo the mountains of southern California: spatial and categorical resolution. In: Quattrochi DA, Goodchild MF, editors. Scale remote Sens. GIS. Boca Raton: CRC Lewis; 1997. p. 3-26.

17. Baldwin DJB, Weaver K, Schnekenburger F, Perera AH. Sensitivity of landscape pattern Índice s to input data characteristics on real landscapes: implications for their use in natural disturbance emulation. Landsc Ecol. 2004;19:255-71.

18. Bailey D, Herzog F, Augenstein I, Aviron S, Billeter R, Szerencsits $\mathrm{E}$, et al. Thematic resolution matters: indicators of landscape pattern for European agro-ecosystems. Ecol Indic. 2007;7:692-709.

19. Buyantuyev A, Wu J. Effects of thematic resolution on landscape pattern analysis. Landsc Ecol. 2007;22:7-13.

20. O'Neill RV, Johnson AR, King AW. A hierarchical framework for the analysis of scale. Landsc Ecol. 1989;3:193-205.

21. Turner MG, O'Neill RV, Gardner RH, Milne BT. Effects of changing spatial scale on the analysis of landscape pattern. Landsc Ecol. 1989;3:153-62.

22. Fisher P. The pixel: a snare and a delusion. Int J Remote Sens. 1997;18:679-85.

23. Blaschke T, Hay GJ, Kelly M, Lang S, Hofmann P, Addink E, et al. Geographic object-based image analysis - towards a new paradigm. Isprs J Photogramm Remote Sens Elsevier. 2014;87:180-91.

24. Tatem AJ, Lewis HG, Atkinson PM, Nixon MS. Super-resolution land cover pattern prediction using a Hopfield neural network. Remote Sens Environ. 2002;79:1-14.

25. Lechner AM, Langford WT, Bekessy SA, Jones SD. Are landscape ecologists addressing uncertainty in their remote sensing data? Landsc Ecol. 2012;27:1249-61.

26. Hengl T. Finding the right pixel size. Comput Geosci. 2006;32: 1283-98.

27. European Environment Agency. CORINE Land Cover [Internet]. Commission of the European Communities; 1994. 1-163.

28. Saura S. Effects of minimum mapping unit on land cover data spatial configuration and composition. Int J Remote Sens. 2002;23:4853-80.

29. Lechner AM, Langford WT, Jones SD, Bekessy SA, Gordon A. Investigating species-environment relationships at multiple scales: 
differentiating between intrinsic scale and the modifiable areal unit problem. Ecol Complex. 2012;11:91-102.

30. Cracknell AP. Review article Synergy in remote sensing-what is in a pixel? Int J Remote Sens. 1998;19:2025-47.

31. Anderson JR, Hardy EE, Roach JT, Witmer RE. Land use and land cover classification system for use with remote sensor data. U S Geol Surv, Prof Pap. 1976.

32. Pojar J, Klinka K, Meidinger DV. Biogeoclimatic ecosystem classification in British Columbia. For Ecol Manag. 1987;22:119-54.

33. Cushman SA, Landguth EL. Scale dependent inference in landscape genetics. Landsc Ecol. 2010;25:967-79.

34. Dungan JL, Perry JN, Dale MRT, Legendre P, Citron-Pousty S, Fortin M-J, et al. A balanced view of scale in spatial statistical analysis. Ecography (Cop). 2002;25:626-40.

35. Dungan JL. Toward a comprehensive view of uncertainty in remote sensing analysis. In: Foody GM, Atkinson PM, editors. Uncertain. Remote Sens. GIS. West Sussex: Wiley; 2002.

36. Csillag F, Fortin M-J, Dungan J. On the limits and extensions of the definiton of scale. Bull ESA. 2000;81:230-232.

37. Skidmore AK, Franklin J, Dawson TP, Pilesjö P. Geospatial tools address emerging issues in spatial ecology: a review and commentary on the Special Issue. Int J Geogr Inf Sci. 2011;25:337-65.

38. Marceau DJ, Gratton DJ, Fournier RA, Fortin JP. Remote sensing and the measurement of geographical entities in a forested environment. 2. The optimal spatial resolution. Remote Sens Environ. 1994:49:105-17.

39. Marceau DJ, Hay GJ. Remote sensing contributions to the scale issue. Can J Remote Sens. 1999;25:357-66.

40. Nelson A. Analysing data across geographic scales in Honduras: detecting levels of organisation within systems. Agric Ecosyst Environ. 2001;85:107-31.

41. Moody A, Woodcock CE. Scale-dependent errors in the estimation of land-cover proportions - implications for global land-cover datasets. Photogramm Eng Remote Sensing. 1994;60:585-94.

42. Smith JH, Wickham JD, Stehman SV, Yang L. Impacts of patch size and land-cover heterogeneity on thematic image classification accuracy. Photogramm Eng Remote Sens. 2002;68:65-70.

43. Pontius RG, Thontteh $\mathrm{O}$, Chen $\mathrm{H}$. Components of information for multiple resolution comparison between maps that share a real variable. Environ Ecol Stat. 2008;15:111-42.

44. Corry RC, Nassauer JI. Limitations of using landscape pattern indices to evaluate the ecological consequences of alternative plans and designs. Landsc Urban Plan. 2005;72:265-80.

45. Castilla G, Larkin K, Linke J, Hay G. The impact of thematic resolution on the patch-mosaic model of natural landscapes. Landsc Ecol. 2009;24:15-23.

46. Pascual-Hortal L, Saura S. Impact of spatial scale on the identification of critical habitat patches for the maintenance of landscape connectivity. Landsc Urban Plan. 2007;83:176-86.

47. Rae C, Rothley K, Dragicevic S. Implications of error and uncertainty for an environmental planning scenario: a sensitivity analysis of GIS-based variables in a reserve design exercise. Landsc Urban Plan. 2007;79:210-7.

48. Pontius Jr RG, Connors J. Range of categorical associations for comparison of maps with mixed pixels. Photogramm Eng Remote Sens. 2009;75:963-9.

49. Bruton MJ, Maron M, Levin N, McAlpine CA. Testing the relevance of binary, mosaic and continuous landscape conceptualisations to reptiles in regenerating dryland landscapes. Landsc Ecol. 2015;30:715-28.

50. Kendall MS, Miller TJ, Pittman SJ. Patterns of scale-dependency and the influence of map resolution on the seascape ecology of reef fish. Mar Ecol Prog Ser. 2011;427:259-74.

51. Stoms DM, Davis FW, Cogan CB. Sensitivity of wildlife habitat models to uncertainties in GIS data. Photogramm Eng Remote Sens. 1992;58:843-50.
52. Lawler JJ, O'Connor RJ, Hunsaker CT, Jones KB, Loveland TR, White $\mathrm{D}$. The effects of habitat resolution on models of avian diversity and distributions: A comparison of two land-cover classifications. Landsc Ecol. 2004;19:517-32.

53. Karl JW, Heglund PJ, Garton EO, Scott JM, Wright NM, Hutto RL. Sensitivity of species habitat-relationship model performance to factors of scale. Ecol Appl Ecol Soc Am. 2000;10:1690-705.

54. Kendall MS, Miller TJ. Relationships among map resolution, fish assemblages, and habitat variables in a coral reef ecosystem. Hydrobiologia. 2010;637:101-19.

55. Allen CR. Discontinuities in ecological data. Proc Natl Acad Sci U S A. 2006;103:6083-4.

56. Nash KL, Allen CR, Angeler DG, Barichievy C, Eason T, Garmestani AS, et al. Discontinuities, cross-scale patterns, and the organization of ecosystems. Ecology. 2014;95:654-67.

57. Wheatley M. Domains of scale in forest-landscape metrics: implications for species-habitat modeling. Acta Oecol. 2010;36:259-67.

58. Kendall MS, Miller T. The influence of thematic and spatial resolution on maps of a coral reef ecosystem. Mar Geod. 2008;31:75102.

59. Neel MC, McGarigal K, Cushman SA. Behavior of class-level landscape metrics across gradients of class aggregation and area. Landsc Ecol. 2004;19:435-55.

60. Nelson MD, McRoberts RE, Holden GR, Bauer ME. Effects of satellite image spatial aggregation and resolution on estimates of forest land area. Int J Remote Sens. 2009;30:1913-40.

61. Pearman PB. The scale of community structure: habitat variation and avian guilds in tropical forest understory. Ecol Monogr Ecol Soc Am. 2002;72:19-39.

62. Holland JD, Bert DG, Fahrig L. Determining the spatial scale of species response to habitat. Bioscience. 2004;54:227-33.

63. Jackson HB, Fahrig L. Are ecologists conducting research at the optimal scale? Glob Ecol Biogeogr. 2015;24:52-63.

64. Miguet P, Jackson HB, Jackson ND, Martin AE, Fahrig L. What determines the spatial extent of landscape effects on species? Landsc Ecol. 2015.

65. Curran PJ, Atikinson PM. Issues of scale and optimal pixel size. Spat Stat Remote Sens. 1999;115-33.

66. Van der Meer F. Remote-sensing image analysis and geostatistics. Int J Remote Sens Taylor Francis. 2012;33:5644-76.

67. Fortin MJ, James PMA, MacKenzie A, Melles SJ, Rayfield B. Spatial statistics, spatial regression, and graph theory in ecology. Spat Stat. 2012;1:100-9.

68. Wagner HH, Fortin M-J. Spatial analysis of landscapes: concepts and statistics. Ecology. 2005;86:1975-87.

69. Lookingbill TR, Rocca ME, Urban DL. Focused Assessment of scale-dependent vegetation pattern. Predict Species Habitat Model Landsc Ecol Concepts Appl. 2011. 111-37.

70. Keitt TH, Urban DL, Ecology S, Sep N. Scale-specific inference using wavelets. Ecology. 2012;86:2497-504.

71. Dray S, Legendre P, Peres-Neto PR. Spatial modelling: a comprehensive framework for principal coordinate analysis of neighbour matrices (PCNM). Ecol Modell. 2006;196:483-93.

72. Lecours V, Devillers R, Schneider D, Lucieer V, Brown C, Edinger E. Spatial scale and geographic context in benthic habitat mapping: review and future directions. Mar Ecol Prog Ser. 2015;535:259-84.

73. Cattarino L, Mcalpine CA, Rhodes JR. Spatial scale and movement behaviour traits control the impacts of habitat fragmentation on individual fitness. J Anim Ecol. 2015;168-77.

74. Leslie H, Ruckelshaus MH, Ball IR, Andelman S. Using siting algorithms in the design of marine reserve networks. Ecol Appl. 2003;13:S185-98. 
75. Gottschalk TK, Aue B, Hotes S, Ekschmitt K. Influence of grain size on species-habitat models. Ecol Modell Elsevier BV. 2011;222: 3403-12.

76. Martin Y, Van Dyck H, Dendoncker N, Titeux N. Testing instead of assuming the importance of land use change scenarios to model species distributions under climate change. Glob Ecol Biogeogr. 2013;22:1204-16

77. Liang Y, He HS, Fraser JS, Wu Z. Thematic and spatial resolutions affect model-based predictions of tree species distribution. PLoS One. $2013 ; 8$, e67889.

78. Saura S, Martinez-Millan J. Sensitivity of landscape pattern metrics to map spatial extent. Photogramm Eng Remote Sensing. 2001;67: $1027-36$

79. Liu Y, Wei X, Li P, Li Q. Sensitivity of correlation structure of classand landscape-level metrics in three diverse regions. Ecol Indic. 2016;64:9-19.

80. Lechner AM, Reinke KJ, Wang Y, Bastin L. Interactions between landcover pattern and geospatial processing methods: Effects on landscape metrics and classification accuracy. Ecol Complex. 2013;15:71-82.

81. Coreau A, Martin JL. Multi-scale study of bird species distribution and of their response to vegetation change: a Mediterranean example. Landsc Ecol. 2007;22:747-64.

82. Zuckerberg B, Desrochers A, Hochachka WM, Fink D, Koenig WD, Dickinson JL. Overlapping landscapes: a persistent, but misdirected concern when collecting and analyzing ecological data. J Wildl Manag. 2012;76:1072-80.

83. Pearman PB. The scale of community structure: habitat variation and avian guilds in tropical forest understory. Ecol Monogr. 2002;72:19-39.

84. Kardel F, Wuyts K, Babanezhad M, Vitharana UWA, Wuytack T, Potters G, et al. Assessing urban habitat quality based on specific leaf area and stomatal characteristics of Plantago lanceolata L. Environ Pollut. 2010;158:788-94.

85. Irl SDH, Harter DEV, Steinbauer MJ, Gallego Puyol D, FernandezPalacios JM, Jentsch A, et al. Climate vs. topography-spatial patterns of plant species diversity and endemism on a high-elevation island. J Ecol. 2015;103:1621-33.

86. Unghire JM, Sutton-grier AE, Flanagan NE, Richardson CJ. Spatial impacts of stream and wetland restoration on riparian soil properties in the North Carolina Piedmont. Restor Ecol 2011;19:738-46.

87. Vicente JR, Gonçalves J, Honrado JP, Randin CF, Pottier J, Broennimann $\mathrm{O}$, et al. A framework for assessing the scale of influence of environmental factors on ecological patterns. Ecol Complex. 2014;20:151-6.

88. Dray S, Pélissier R, Couteron P, Fortin MJ, Legendre P, Peres-Neto $\mathrm{PR}$, et al. Community ecology in the age of multivariate spatial analysis. Ecol Monogr. 2012;82:257-75.

89. Mayor SJ, Schneider DC, Schaefer JA, Mahoney SP. Habitat selection at multiple scales. Ecoscience. 2009;16:238-47.

90. Zetterberg A, Mörtberg UM, Balfors B. Making graph theory operational for landscape ecological assessments, planning, and design. Landsc Urban Plan. 2010;95:181-91.

91. Schick RS, Loarie SR, Colchero F, Best BD, Boustany A, Conde DA, et al. Understanding movement data and movement processes: current and emerging directions. Ecol Lett. 2008. 1338-50.

92. Karl JW, Maurer BA. Spatial dependence of predictions from image segmentation: a variogram-based method to determine appropriate scales for producing land-management information. Ecol Inform. 2010;5:194-202.

93. Vicente J, Randin CF, Goncalves J, Metzger MJ, Lomba A, Honrado J, et al. Where will conflicts between alien and rare species occur after climate and land-use change? A test with a novel combined modelling approach. Biol Invasions. 2011;13:1209-27. 